\title{
Fertility preservation options for transgender individuals
}

\author{
Joshua Sterling ${ }^{1}$, Maurice M. Garcia ${ }^{2,3,4}$ \\ ${ }^{1}$ Division of Urology, Rutgers Robert Wood Johnson Medical School, New Brunswick, NJ, USA; ${ }^{2}$ Division of Urology, Cedars-Sinai Medical Center, Los \\ Angeles, Los Angeles, CA, USA; ${ }^{3}$ Department of Urology, ${ }^{4}$ Department of Anatomy, University of California San Francisco, San Francisco, CA, USA \\ Contributions: (I) Conception and design: All authors; (II) Administrative support: MM Garcia; (III) Provision of study material or patients: All \\ authors; (IV) Collection and assembly of data: J Sterling; (V) Data analysis and interpretation: All authors; (VI) Manuscript writing: All authors; (VII) \\ Final approval of manuscript: All authors. \\ Correspondence to: Maurice M. Garcia, MD, MAS. Director, Transgender Surgery and Health Program; Division of Urology, Cedars-Sinai Medical \\ Center, 8635 W. Third St. Suite 1070 W., Los Angeles, CA 90048, USA. Email: Maurice.garcia@cshs.org.
}

\begin{abstract}
Gender affirming medical and surgical treatments affect the reproductive potential of transgender individuals. Prior to the development of assisted reproductive technologies (ART), genital gender-affirming surgery frequently eliminated a patient's reproductive potential. Today, all patients should be counseled on their fertility preservation (FP) options before medical and surgical transition, yet this appears to seldom occur in practice. The following review is the result of a systematic literature search of PubMed, Medline and Google Scholar to identify current and future FP options, barriers to treatment patients face, practice patterns of transgender health care providers, and if there were any standardized counseling protocols. Options for transwomen at any point in their transition range from simply providing a semen sample to be used with assistive reproductive techniques to experimental techniques involving testicular cryopreservation followed by in vitro initiation of spermatogenesis. Transmen before and after starting hormone therapy can pursue any assistive reproductive techniques available for ciswomen. Future options currently under investigation include ovarian tissue cryopreservation (OTC) with in vitro oocyte maturation. In addition to counseling about their FP options, patients should be advised prospectively about the requirements, process details, the total costs associated with achieving pregnancy, and the inherent risks associated with using preserved genetic material including risk of failure, and maternal and fetal health risks. Transgender patients report using assistive reproductive services difficult, due to a lack of dialogue about fertility and the lack of information offered to them- presumably because their circumstances do not fit into a traditional narrative familiar to providers. Physicians and health care providers would benefit from better educational tools to help transgender patients make informed decisions and better training about transgender patients in general, and FP options available to them.
\end{abstract}

Keywords: Fertility preservation (FP); trans male; trans female; transgender health; assistive reproductive technologies

Submitted Jun 25, 2019. Accepted for publication Sep 17, 2019.

doi: $10.21037 /$ tau.2019.09.28

View this article at: http://dx.doi.org/10.21037/tau.2019.09.28

\section{Introduction}

In most areas of clinical medicine where treatments can impact a patient's future fertility options fertility preservation (FP) is routinely discussed. This otherwise standard practice, however, is lacking in the care of transgender people. Historically, it was believed that gender dysphoria should always preclude the individual from having a biologic child. These views may have been based on a pathologized view that saw being transgender as a disorder instead of an example of natural human diversity, wherein procreation should be prevented. As social norms and attitudes have evolved, however, the basis for these positions has been 
debunked, and antiquated laws requiring that genital gender-affirming surgery result in permanent sterilization are being challenged in the courts and repealed (1). The World Professional Association for Transgender Health (WPATH) Standards of Care for the Health of Transsexual, Transgender, and Gender Nonconforming People (SOC) version 7 [2012], for the first time included a section on reproductive health, which recommended that counseling on FP be provided for all transgender individuals (2).

There are no accepted ethical issues that would preclude FP in transgender individuals, and data shows that being a transgender parent, by itself, has no negative impact on a child's upbringing, whether the parent's transition occurred before or after the child was born (3). However, societal prejudices that trans individuals are poor parents, social stigma associated with being a transgender parent, and general fears that the child would be ostracized are often mentioned by transgender individuals, adults and adolescents, as a reason why they do not envision being the biologic parent of a child (4). We have found this fear to be voiced in FP discussions with our patients. Recent media coverage of transgender individuals and transgender discrimination including fertility considerations has increased public consciousness of these issues worldwide. Goldman et al. surveyed over 1,000 Americans and found that over $90 \%$ of responders either agreed with or were neutral to doctors providing fertility care to transgender individuals (5). The knowledge that FP in transgender individuals is supported by a majority of Americans may help members of the transgender community access any desired fertility care. When patients are considering FP they should weigh the personal benefit of future biologic children with the risks of any procedures, and possibility of worsening gender dysphoria (6).

The aim of this review is to analyze the growing body of literature regarding FP in transgender patients. We discuss both current and future options allowing trans people to have their own biologic offspring, highlight the barriers limiting access to this basic right, and examine current practice patterns of physicians and health care providers specializing in transgender health.

We performed a systematic search of PubMed, Google Scholar and Medline, using all iterations of the following search terms: transgender, gender non-conforming, gender dysphoria, transsexual, female to male, male to female, transman, transwoman, infertility, fertility, FP, cryopreservation, sperm banking, egg banking, assistive reproductive techniques, and all acceptable abbreviations.
Given the limited amount of existing literature, inclusion was broad. After eliminating duplicates and abstracts, all queries yielded 81 unique publications, of various types including proposed guidelines, commentaries, other reviews, case series, qualitative, and quantitative research. Publications were then grouped based on topic (ethical and legal considerations, patient-focused, provider focused, FP options) and reviewed for relevance.

\section{FP options}

For all transgender individuals, fertility options can currently be categorized into three different groups: (I) options available before initiation of gender-affirming hormone therapy (TT); (II) options available after initiation of GAHT; and (III) experimental options, which can be done concurrently with genital gender-affirming surgery. Fertility options are summarized in Tables 1 and 2. The reproductive changes in patients taking GnRH agonists for precocious puberty have been shown to be reversible in males and females $(7,8)$. However, there have been no studies on the effect of GnRH agonists on a patient's native gametes/gamete-producing organs when these are used concurrently with GAHT. Currently, there is no published data concerning viability of a patient's native gametes following long term GAHT use. Due to this lack of information it is not possible to say what the FP options are for individuals that transition before puberty. However, the experimental options discussed have the potential applications in this population but will require further development and advances in gamete maturation techniques after tissue cryopreservation before they can be discussed as realistic options (9).

While the FP options for pre-pubertal and early adolescent patients are experimental at this time, they should get the same consideration as adults. These patients are making medical decisions, and the American Academy of Pediatrics bioethics guidelines state pediatric patients who are judged to have decisional capacity are entitled to the same dress of autonomy as adult patients (6). Half of transgender youths agreed that their feelings about wanting a biologic child might change in the future. The relatively higher incidence of mental health co-morbidities (e.g., depression and anxiety) in transgender adolescents (as compared to their cisgender counterparts) may impact their ability to consider and process considerations about their future and future needs $(2,10,11)$. This patient uncertainty and limited scientific evidence make it more important 
Table 1 Fertility preservation options for transwomen

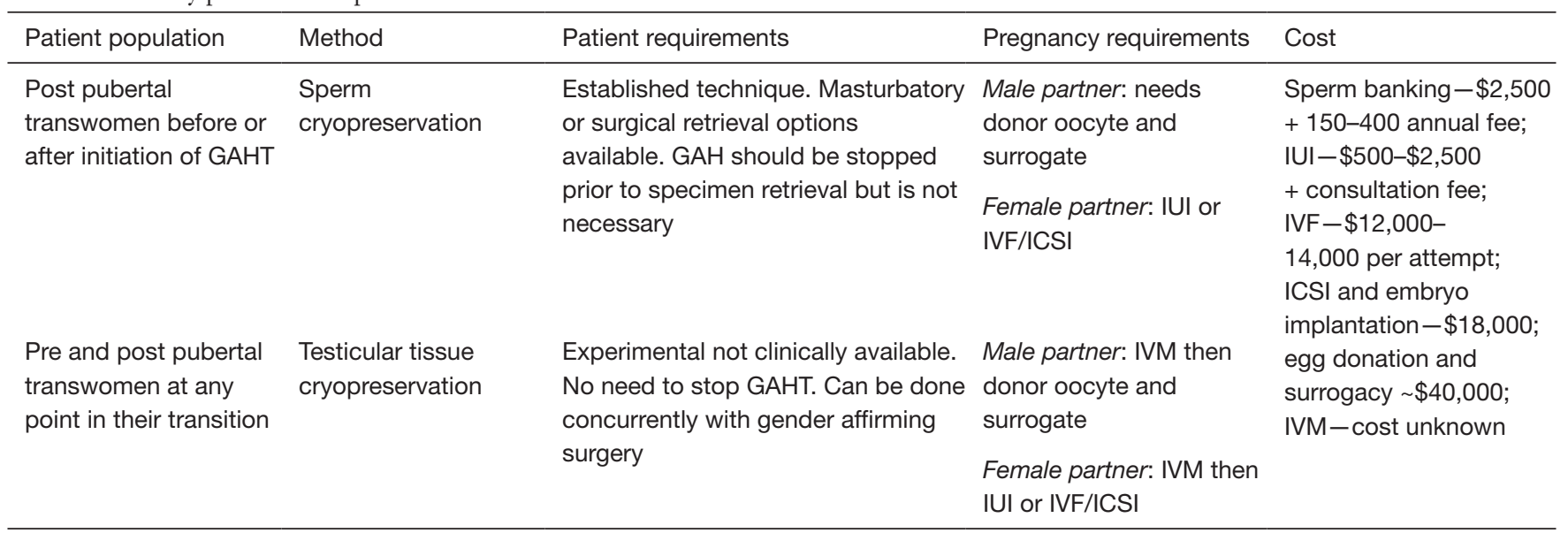

IUI, intrauterine insemination; IVF, in vitro fertilization; ICSI, intracytoplasmic sperm injection; IVM, in vitro maturation.

to have open and honest conversations. FP discussions with children and adolescents should include parents, but it should primarily focus on maintaining the patient's autonomy.

\section{Options for transwomen: before initiation of GAHT}

For post-pubertal transwomen, the simplest method of FP is the cryopreservation of a sample of ejaculated semen. However, for many transwomen, the focused-use of their genitals can generate significant dysphoria. Also, it is possible to use a partner for stimulation and simple masturbatory efforts, samples can also be procured with the assistance of vibratory stimulation or electroejaculation, though the latter is painful and typically requires general anesthesia. Samples can later be used with assisted reproductive technologies (ART) (12). Alternatively, transwomen who find it distressing to produce an ejaculated sample by the above methods can undergo sperm aspiration or microsurgical sperm extraction, which is a more invasive and expensive retrieval approach. Small studies from Europe, where sperm banking and ART are more accessible to patients than in the U.S. have reported that 55-66\% of trans women who identify as lesbian or bisexual have frozen their sperm compared to only $13 \%$ of heterosexual transwomen $(13,14)$, though these findings or trends cannot be generalized to U.S. centers. While there is little structured exploration of the greater likelihood for patients with female partners to be more likely to bank sperm than patients with only male partners, it is likely related to a greater probability that bisexual and lesbian transwomen already have an intimate partner at the time of evaluation, with whom to use the preserved specimen and carry the pregnancy.

\section{Options for transwomen: after initiation of GAHT}

There is limited evidence describing semen characteristics of transwomen or the success rates of ART using specimens from transwomen. Transwomen who are on GAHT can undergo sperm cryopreservation, but there is greater uncertainty of the viability of their samples. Two studies, Hamada et al. and Li et al., reported poor semen quality (decrease volume, sperm count, and motility) of cryopreserved samples from transwomen compare to accepted standards or matched cisgender controls $(15,16)$. Both studies are limited by not having data regarding hormone status of the donating individuals. It stands to reason that semen parameters might be negatively affected by GAHT, but only one published study investigated this question. Adeleye et al. analyzed semen samples from 28 patients and found that specimens collected during concurrent GAHT were associated with abnormal parameters. However, samples collected after cessation of GAHT, with a mean discontinuation time of 4.4 months, were comparable to those from individuals who had yet to start GAHT (17). This was a very small sample size, but cessation of GAHT seems to reverse the negative effects of estrogen on spermatogenesis. It remains unclear how long hormone treatments need to be stopped before testicular histology, and semen parameters return to normal (18). In cis-men the process starting with spermatogenesis and 
Table 2 Fertility preservation options for transmen

\begin{tabular}{|c|c|c|c|c|}
\hline Patient population & Method & Patient requirements & Pregnancy requirements & Cost \\
\hline $\begin{array}{l}\text { Post pubertal } \\
\text { transmen before } \\
\text { and after initiation of } \\
\text { GAHT }\end{array}$ & $\begin{array}{l}\text { Oocyte } \\
\text { cryopreservation }\end{array}$ & $\begin{array}{l}\text { Established practice. Should } \\
\text { stop GAHT and undergo } \\
\text { controlled ovarian stimulation } \\
\text { with transvaginal oocyte } \\
\text { retrieval }\end{array}$ & $\begin{array}{l}\text { Male partner: use partners } \\
\text { sperm for fertilization. Need } \\
\text { surrogate to carry embryo to } \\
\text { term } \\
\text { Female partner: sperm donor } \\
\text { for fertilization, transplantation } \\
\text { of embryo into partners uterus }\end{array}$ & $\begin{array}{l}\text { Egg freezing }-\$ 8,000-12,000 \\
+\$ 500 \text { annual fee; } \\
\text { IVF and embryo } \\
\text { transfer }-\$ 12,000-14,000 ; \\
\text { Surrogacy fees }-\sim 30,000\end{array}$ \\
\hline
\end{tabular}

IVM, in vitro maturation.

resulting with a mature sperm able to produce a pregnancy requires approximately 3 months, and for this reason, a reasonable guideline to avoid any adverse effects from GAHT upon sperm quality is to recommend cessation of GAHT for at least 3 months before retrieving sperm from semen (19). During this break in treatment, resuming normal testosterone levels may result in unwanted masculinizing effects, which adds an emotional cost to the already substantial price of FP.

\section{Experimental FP options for transwomen}

For patients that are unable to produce a semen specimen, the use of cryopreserved testicular tissue from which to grow and mature sperm ex vivo may be an option in the future. While cryopreservation of testicular tissue is possible now, its use to create healthy sperm capable of achieving pregnancy remains experimental. With this procedure, testicular tissue is harvested at time of the orchiectomy and is cryopreserved. Later, in a controlled in vitro environment, the testicular tissue is exposed to hormonal and cell-culture agents to trigger spermatogonial stem cells to restart spermatogenesis $(20,21)$. The approach of in vitro growth and differentiation of germ cells able to fertilize an egg has been validated using a mouse model (22), but not in a human model (23). Apart from the practical (techniquebased) challenges, another source of concern is the unknown quality of gametes matured and developed ex vivo. Ex vivo conditions in general, and/or some of the hormonal and culture-media agents used in particular, could change/ alter sperm in unforeseen ways. It has been shown that ART , specifically techniques like ICSI which bypass the natural barriers preventing unfit sperm from fertilizing an egg, is associated with a higher rate of birth defects and this risk could be further multiplied using gametes matured in an ex vivo environment (24-26).

It should be noted that when this technique is used for pre-pubertal patients, the testicle tissue used as substrate for spermatogenesis is itself also immature, which may make this technique more challenging and/or introduce greater risk of genetic abnormalities in the resulting sperm unless natural maturational tissue changes associated with puberty are controlled for during ex vivo culture. The transition into puberty may, for tissues and gametes, be a natural window 
for cell quality control. Nonetheless, an exciting promise of ex vivo spermatogenesis, if/when it is ever validated and becomes a clinical option for patients, is this approach would afford FP for adults who are unable to produce a semen sample without an additional procedure and provide an FP option for transgender patients who start their transition prior to the natural onset of puberty.

Another promising avenue for FP for transwomen is uterine transplantation. To date, however, this has only been attempted in ciswomen. A Swedish research group reported the first live birth using a transplanted uterus in 2014 and was subsequently performed successfully for the first time in the U.S. in 2017 (27,28). For trans women, however, there are several anatomic and technical hurdles that must be addressed before uterine transplant could be attempted. These include creation of a vaginal outlet both for egress of uterine discharge/waste, and, for reliable access to the uterus for routine biopsies and monitoring. These challenges are currently being explored in cadaveric and animal models (29).

\section{Options for transmen: before and after Initiation of GAHT}

A transman can carry a pregnancy to term or pursue FP via cryopreservation of oocytes or embryos both before and after initiation of GAHT (30,31). Light et al. surveyed 41 transmen in the U.S. who became pregnant and carried to term after they had transitioned socially and/or medically. $61 \%$ reported prior testosterone use and $68 \%$ were planned pregnancies. There were no differences in perinatal complications, delivery or birth outcomes based on prior testosterone use. Patient responses to how pregnancy impacted their gender dysphoria ranged from significantly improved to worsened (32). Almost half of the pregnancies in individuals who had no previous testosterone use and $24 \%$ of pregnancies in patients with prior testosterone use were unplanned and conceived with concurrent Testosterone therapy, which highlights the need to discuss contraceptive methods with transmen (32).

While the literature reports amenorrhea commonly occurs 6 months after initiation of GAHT, there is no agreement on the timing of resumption of menses after cessation of testosterone $(33,34)$. Small studies have suggested the majority of patients resume menstruation within 6 months of stopping GAHT, but the small sample size and varied duration of GAHT prior to cessation make this finding hard to generalize (32). There are no studies looking recovery of menstruation after long term GAHT or how prepubertal suppression and concurrent GAHT affect fertility. Cryopreservation of both oocytes and embryos is a clinically established FP method in cisgender women but has several additional considerations for transgender men. In addition to stopping GAHT for an undetermined amount of time, transmen considering ART must also be made aware that they will need to undergo the following during the course of preparation for and completion of, pregnancy: routine transvaginal ultrasound examinations, ovarian stimulation with exogenous administration of female hormones, and finally an invasive transvaginal procedure to harvest the oocyte and to assess embryo or uterine health. Some or all of these procedures can be very distressing to transmen $(9,31)$. Small case series have reported live births following cryopreservation of oocytes and embryos in patients with a history of testosterone use as well as those who underwent FP prior to GAHT $(9,20,35,36)$. In cis-women birth rates using frozen oocytes are comparable to birth rates using fresh oocytes, which eliminates the need to fertilize the oocyte at time of harvest and affords the option of deferring procurement of a partner and sperm to a later date. However, given the small numbers of live births using ART in transmen we are unable compare birth rate outcomes using fresh vs frozen oocytes in this patient population.

\section{Experimental FP options for transmen}

In instances where the rise in gender incongruent hormones makes ovarian stimulation unacceptable, ovarian tissue cryopreservation (OTC) may be a viable option for transgender men in the future. The procedure for OTC involves cryopreservation of the whole ovary or an ovarian cortex biopsy, then thawing and maturing the immature follicles later to be used with established ARTs. In transmen OTC would avoid the unwanted effects of ovarian stimulation, patients do not have to stop GAHT, and it does not require additional procedures as cryopreservation can be done concurrently with gender-affirming surgery $(21,37)$. This option remains in the developmental phase while the method to mature the immature follicles is being perfected. Maturation is achieved in cis-women via reimplantation of the ovarian cortical tissue into the patient, which results in resumption of the normal female hormonal cycle and natural maturation of the immature oocytes. To date over 100 live births have been reported following auto-transplantation of thawed ovarian tissue; orthotopic reimplantation in the pelvis is more common and more successful than use of a heterotopic site (ex: arm 
or abdominal wall) (38-41). However, this method would be problematic for transmen who do not want restoration of female hormonal cycles and maybe impossible for transmen who started their transition prepubertally.

An alternative solution may be in vitro maturation (IVM) of the immature gametes $(37,42,43)$. IVM is a process of maturation in culture of immature oocytes that had not been exposed to luteinizing hormone or human chorionic gonadotropin prior to retrieval to induce meiotic resumption. During the process immature oocytes progress from meiosis I to metaphase II (44). De Roo et al. demonstrated that IVM following OTC in transmen, who had not stopped GAHT prior to oophorectomy, was possible. Spindle analysis found a normal chromosomal pattern in $87 \%$ of the metaphase II oocytes obtained (37). However, achievement of metaphase II does not equate the ability for successful fertilization. There are cytoplasmic changes necessary for fertilization and embryonic development that are difficult to assess microscopically. To date successful fertilization and embryo implantation following IVM of an immature oocyte from a transman has not been reported.

\section{Patient barriers}

\section{Poor utilization due to societal barriers}

Studies of transgender patients in America, Australia, Canada, and Germany found that the majority had a desire for a family and $15-24 \%$ were already parents, but only a small percentage, $<10 \%$, undergo FP or ART (45-48). Of the 409 patients surveyed in Riggs et al. 77\% were not given any advice or counseling about their FP options, and thus only $7 \%$ had undertaken any sort of FP $(46,47)$. Auer et al. found $35 \%$ of respondents wanted genetically related children, but only $9 \%$ of transwomen report sperm freezing. No transmen reported oocyte cryopreservation despite $15.4 \%$ of pre-treatment individuals and $12.5 \%$ of those that on GAHT reporting oocyte preservation was an acceptable method of FP (48). It should be noted that egg donation is illegal in Germany prohibiting transmen from using cryopreserved oocytes with a future partner.

It should be noted that the aforementioned studies were conducted in countries with socialized healthcare systems that afford patients greater access to ART than in the U.S. Greater access to and/or affordability of ART can reasonably influence patient-reported preferences and utilization of ART. Limitations on access to ART, such as occurs in the
U.S. due to high out-of-pocket costs for patients, can also confound understanding of alternative reasons for why some patients, despite being offered ART for FP, may not wish to pursue FP. In our own clinical experience (MMG), a subset of patients who desire to be parents decline FP in favor of adoption due to an ambivalence of passing their own genes, and any possible genetic component to being transgender, to their children. Hence, we believe that it is also important to explain to patients, in a way that does not pathologize being transgender, that to date, there is no known genetic component to being transgender.

\section{Lack of counseling and information}

Laws that once required sterilization as a part of a patient's transition have been repealed, but there are still barriers preventing access to care. WPATH current standards of care recommend all transgender individuals be given information and counseling regarding FP, yet most individuals do not receive counseling at any point in their transition process (46-49). Those that are made aware of their options and express interest in FP are often left to their own efforts to identify and understand the next steps in the FP process. This lack of guidance can leave patients frustrated and confused, as prenatal care facilities/programs, midwife centers, and ART facilities are often unprepared to serve transgender patients seeking FP and perinatal care (50).

A recent small study looked at the experiences of trans individuals who sought or utilized assistive reproductive services in Ontario between 2007 and 2010, and only two of the nine individuals interviewed had a positive interaction (51). The negative interactions could be categorized into three groups: (I) 'Problems with clinical documentation,' (II) 'Impact of providers cis-normative and hetero-normative assumptions, and (III) 'Refusal of services.' One of the individuals interviewed suggested their issue may have been due to discrimination on the basis of gender identity, but most patients reported that the issues they faced were based on poor communications and a lack of mutual understanding with their providers. Patients reported feeling alienated by forms and documentation that did not allow them to express their specific identity and misunderstood by health care providers that didn't take the time to understand their unique situations and needs. Of those interviewed the best encounter occurred when the endocrinologist managing the patient's GAHT was also a fertility specialist and thus well educated about trans-related 
health care (51). Patients that have already undergone medical or surgical transition have reported a wishing they received some FP counseling, but there is currently no research on the rate of decisional regret in transgender patients that experience infertility (48).

The absence of information is compounded in the adolescent population because until recently, the literature concerning FP in adolescent transgender individuals focused on ethical instead of substantive discussions. Chen et al. surveyed 156 adolescents ages 14-17 and found 44\% of gender-nonconforming youth and $26 \%$ of transgender youth expressed interest in biological fertility but reported that discussions about fertility and FP options with healthcare providers were uncommon. Only $20 \%$ discussed fertility in general, and $13 \%$ discussed the effects of genderaffirming hormones on fertility (52). When surveyed almost youths and parent considered it important to learn how hormone therapies could impact fertility (10). The most common source for information reported by patients and their families was the internet, but only $16 \%$ Society for Assistive Reproductive Technologies (SART) member websites contained information concerning transgender patients (53).

\section{Cost of FP}

Cost, by itself, was also an overwhelmingly significant barrier in countries like the U.S. where there is little to no financial assistance for FP. Google search of cost of ART available in the U.S. varied greatly depending on regional location, but the average costs are as follow here. For a trans woman seeking to pursue sperm banking in the U.S. there is an initial cost of $\sim \$ 2,500$, which usually includes the storage fee for the first year, and then the annual storage cost ranges from $\sim 150$ to $\$ 400$, depending on facility. The cost of harvesting and freezing eggs is significantly higher, with initial costs ranging from $\$ 8,000$ to $\$ 12,000$ plus a $\sim 500$ annual storage fee. Then, because the use of preserved gametes will always require some ART, there are always additional (and often relatively high) out of pocket costs.

\section{Cost to achieve pregnancy and live birth}

At the less expensive end the cost spectrum, we found that intrauterine insemination ranges from $\$ 500$ to $\$ 2,500$ per attempt. Each cycle of IVF costs between $\$ 12,000$ to $\$ 14,000$, and that cost can increase to almost $\$ 20,000$ if ICSI is utilized to fertilize the egg. These costs can quickly add up to tens of thousands of dollars. These costs can be especially daunting for adolescent individuals who are not financially independent and who are also often many years away from considering any family planning options. However, recent surveys of adolescent transgender patients suggest that while cost is a consideration for FP, it is not necessarily the most important in comparison to other unique considerations reviewed below $(4,10,52)$.

\section{Provider barriers}

Transgender adults and adolescents vary in their desire for parenthood. Past notions that trans individuals don't desire a family, or that the loss of biologic fertility should be a prerequisite to gender transition have been thoroughly discredited. Studies and our own experience suggest that most transgender patients and their family members report a desire for better education and counseling regarding fertility options, which they often do not receive in conversations with their physicians.

Only two studies assessed practice patterns of different health care professionals caring for transgender people. Tishelman et al. surveyed 255 health care providers from 9 different countries representing four types of providers: physicians, psychologists, master-level mental health providers, and physician extenders. Multiple barriers were identified across the various responders, including structural factors (access to care and cost), patient factors (urgency for gender-affirming treatment), and provider factors (uncertainty about counseling roles, and general lack of information in this nascent field) (54). There are currently no guidelines addressing the specific complexities of this issue such as actual fertility risks of gender-affirming hormone therapy, optimal counseling techniques based on patient age or stage in the transition process to assist providers that are motivated to appropriately counsel patients and families.

Chen et al. created a 46-item survey targeted for providers in transgender healthcare to assess baseline knowledge, practice patterns, and perceived barriers to fertility care. While most providers were sufficiently knowledgeable about the impact of GAHT on fertility, they felt much less confident in their knowledge surrounding FP. Baseline knowledge of fertility issues was comparable among physicians, psychologists and physician extenders, but it was a topic only physicians frequently discussed with patients (55). The most common reason given for this gap was specific perceptions of roles and specific gaps in knowledge related to FP. Many psychologists and physician 
extenders felt their role was to openly discuss any issues with patient but refer the patient to a more qualified party like a fertility specialist to discuss specifics. Physicians report feeling inadequately prepared to counsel patients due to a lack of scholarly publications on best practices, success rates and rate of decisional regret in patients who do not purse FP (55). Providers also reported not wanting to bring up FP discussions multiple time due to fear that they may be construed as pushing cis-normative and hetero-centric bias on patients $(54,55)$. This often leaves patients without a clear treatment path even if they were interested in FP. This also highlights the importance of providers facilitating an environment of trust and acceptance with their transgender patients, where discussion topics can be more freely touched upon and pursued by both patient and provider.

When FP is discussed, many patients report being given overly general information before then being referred to fertility specialists for a more thorough discussion, but, only $16 \%$ of SART member clinics have any publicly available information related to transgender individuals (53). The need for transgender decision aids is well reported, but currently no standardized tools have been developed (56). There is ample evidence in the oncofertility literature reporting that inconsistent counseling practices resulted in low FP utilization and that by standardizing counseling practices and providing education session for physicians on FP more patients opted to undergo FP and overall patient satisfaction increased (57-60). Transgender health care providers and patients would both benefit from the development of standardized informational materials and counseling protocols regarding FP options.

\section{Limitations of in vitro fertilization (IVF)}

Patients considering assisted reproduction should be aware of the principal limitations with ART procedures $(24,61,62)$. IVF is associated with limitations in three critical domains: success rate (defined as live births per the number of embryos transferred), morbidity (health risks to mother and fetus arising from multiple-birth gestation), and cost (to patient, and health-care system) $(26,63)$.

Success: in 2015 the overall IVF delivery rate per cycle was $33 \%$, and the likelihood of success decreased significantly after the fourth cycle (64). Historically, due to the relatively low success rate of IVF, an average of 2-3 embryos are typically transferred to the mother per cycle; this results in a high multiple-birth rate. However, the rate of higher-order pregnancies has dropped dramatically over the last decade as single embryo transfer became more common. In 2015, the rate of carrying three or more fetuses was $<1 \%$; the rate of twins remained relatively high (22\%) (64).

Morbidity/risk: a multiple-birth pregnancy is the single greatest source of morbidity and mortality to both mother and fetus, as these are closely associated with prematurity, low birth weight, Caesarian section, and, for both mother and fetus, increased risk of prolonged hospital stay, disability, or death $(25,63,65)$. With intracytoplasmic sperm injection (ICSI), sperm selection is operator dependent, and as such, bypasses the process of 'natural selection' to determine which individual sperm fertilizes the oocyte $(24,61)$. Inadvertent use of dead or dying sperm or poorquality oocytes likely contributes to unfavorable outcomes, such as immediate or delayed embryo failure, and genetic mutations and/or epigenetic effects in the developing embryo potentially resulting in an increased incidence of birth defects $(25,26,63,65-67)$.

Cost: in the U.S., the average out of the pocket cost of an IVF cycle ranges from $\$ 10-\$ 15,000$ depending on insurance coverage, patient-specific factors, and treatment center; the average number of cycles per live birth is $>3$ (64). Currently, fourteen states [Arkansas, California, Connecticut, Hawaii, Illinois, Maryland, Massachusetts, Montana, New Jersey, New York, Ohio, Rhode Island, Texas, and West Virginia] require insurers to cover some form of infertility treatment, but the scope of what is covered varies greatly (64). Patients need to contact their insurance provider to see exactly what fertility treatments are covered and what costs will be out of pocket. Poor outcomes with respect to these three domains (success rate, morbidity, and cost) are rooted, at least in part, to our inability to reliably predict which 1-2 embryos, produced in vitro, is likeliest to result in a live birth following transfer to the uterus (62).

\section{Discussion and conclusions}

We found that despite a reported high interest in FP, there was a very low utilization rate. The most common reasons given for this discrepancy are lack of opportunity to discuss FP options with their providers, lack of reliable information available from other and outside sources, and the considerable out of pocket costs. Current FP counseling practices are infrequent occurrences where FP is discussed in abstract terms and the all-in costs from FP to ART to delivery of a healthy newborn are rarely mentioned, and then patients are referred to fertility clinics which are often untrained and ill-equipped to deal with the unique 
Table 3 Key takeaway points

Most patients report interest in FP but only $10 \%$ end up pursuing FP

Patients report lack of information, miscommunication and cost as main reasons for not pursuing FP

Transgender healthcare providers feel ill-prepared to discuss FP and most fertility clinics don't understand the unique psychologic and physiologic realities of transgender patients

Standardized patient education materials and provider training have been shown to increase overall patient satisfaction and increase FP utilization in other patient populations and could provide a model for transgender patients

Transgender individuals have FP options at any/all points during their transition (before GAH, after GAH, at time of confirmation surgery) but risks, outcomes, and present and future costs are not the same, and options warrant discussion with an informed provider. Costs, requirements, and risks associated with use of preserved cells (i.e., future events) should be especially clear

To deal with this uncertainty it is important to cultivate an environment of trust, and knowledgeable, culturally sensitive providers to whom to refer patients - where topics can be discussed freely and honestly

FP, fertility preservation.

psychologic and physiologic differences and challenges that many transgender patients may present for providers not familiar with trans care. Our findings, summarized in Table 3, clearly suggest that health care providers must improve access to and quality of counseling for patients about FP to transgender patients.

Standardized patient education materials should be developed so that patients are aware of all FP options available at every stage of their transition, what the cost of preservation is, and all of the steps and potential challenges that FP, resulting in a successful live birth, requires. Though outside the scope of this review, we find for many patients, it would be useful to them to have access to statespecific rules and regulations related to adoption, as many patients are interested in this. Educational materials are also needed specifically for health care providers so that they feel comfortable and capable of answering fertility-related questions transgender individuals may have at various points along their transition process. The success of FP counseling and training programs adopted for oncology patients and providers can serve as a model in the area of transgender health.

Parents should be involved in FP discussions with early adolescent and pre-pubertal patients, but all discussion should also include a focus on maintaining the patient's autonomy. It is important to stress that there is no evidence about how long-term GAHT affects fertility or the effect of combined use of GnRH agonists with GAHT on a patient's native gonadal tissues. Currently all FP options for prepubertal and early adolescent patients are experimental and require several scientific advances before they have clinical relevance.
Transgender individuals have the option for FP at any point in their transition, including tissue preservation at time of orchiectomy or oophorectomy. Banking of gametes does not guarantee access to or success of future IVF treatments; success depends on the technique used and the quality of the specimen from both parties. Medically assisted reproduction is considered to be safe but is not without maternal and fetal health risks (24-26). The increased use of ART in transgender patients raises the need for adaptive healthcare strategies before fertilization, during pregnancy, and after birth.

In conclusion, fertility and FP in transgender individuals is an area of great clinical importance and active research. Patients should be counseled on reproductive issues by professionals. However, best practices on when and how often this counseling should occur have yet to be established. It is also important to develop clear and detailed information so that patients, family members, and partners can make a well-informed decision regarding future family planning.

\section{Acknowledgments}

Funding: None.

\section{Footnote}

Provenance and Peer Review: This article was commissioned by the Guest Editors (Larry I. Lipshultz, Alexander W. Pastuszak) for the focused issue "Contemporary Issues and Controversies in Men's Health" published in Translational Andrology and Urology. The article was sent for external peer 
review organized by the Guest Editors and the editorial office.

Conflicts of Interest: The focused issue "Contemporary Issues and Controversies in Men's Health" was commissioned by the editorial office without any funding or sponsorship. The authors have no conflicts of interest to declare.

Ethical Statement: The authors are accountable for all aspects of the work in ensuring that questions related to the accuracy or integrity of any part of the work are appropriately investigated and resolved.

Open Access Statement: This is an Open Access article distributed in accordance with the Creative Commons Attribution-NonCommercial-NoDerivs 4.0 International License (CC BY-NC-ND 4.0), which permits the noncommercial replication and distribution of the article with the strict proviso that no changes or edits are made and the original work is properly cited (including links to both the formal publication through the relevant DOI and the license). See: https://creativecommons.org/licenses/by-ncnd/4.0/.

\section{References}

1. Dunne P. YY v Turkey: infertility as a pre-condition for gender confirmation surgery. Med Law Rev 2015;23:646-58.

2. World Professional Association for Transgender Health EMRWG. Standards of care for health of transsexual, transgender, and gender nonconforming people, 7 th version 2011.

3. Murphy TF. The ethics of fertility preservation in transgender body modifications. J Bioeth Inq 2012;9:311-6.

4. Chiniara LN, Viner C, Palmert M, et al. Perspectives on fertility preservation and parenthood among transgender youth and their parents. Arch Dis Child 2019;104:739-44.

5. Goldman RH, Kaser DJ, Missmer SA, et al. Fertility treatment for the transgender community: a public opinion study. J Assist Reprod Genet 2017;34:1457-67.

6. Chen D, Simons L. Ethical Considerations in Fertility Preservation for Transgender Youth: A Case Illustration. Clin Pract Pediatr Psychol 2018;6:93-100.

7. Bertelloni S, Baroncelli GI, Ferdeghini M, et al. Final height, gonadal function and bone mineral density of adolescent males with central precocious puberty after therapy with gonadotropin-releasing hormone analogues.
Eur J Pediatr 2000;159:369-74.

8. Pasquino AM, Pucarelli I, Accardo F, et al. Long-term observation of 87 girls with idiopathic central precocious puberty treated with gonadotropin-releasing hormone analogs: impact on adult height, body mass index, bone mineral content, and reproductive function. J Clin Endocrinol Metab 2008;93:190-5.

9. Moravek MB. Fertility preservation options for transgender and gender-nonconforming individuals. Curr Opin Obstet Gynecol 2019;31:170-6.

10. Strang JF, Jarin J, Call D, et al. Transgender Youth Fertility Attitudes Questionnaire: Measure Development in Nonautistic and Autistic Transgender Youth and Their Parents. J Adolesc Health 2018;62:128-35.

11. Nahata L, Curci MB, Quinn GP. Exploring Fertility Preservation Intentions Among Transgender Youth. J Adolesc Health 2018;62:123-5.

12. De Roo C, Tilleman K, T'Sjoen G, et al. Fertility options in transgender people. Int Rev Psychiatry 2016;28:112-9.

13. De Sutter P, Verschoor A, Hotimsky A. The desire to have children and the 101 preservation of fertility in transsexual women: A survey. Internation Journal of Transgenderism 2002;6.

14. Wierckx K, Stuyver I, Weyers S, et al. Sperm freezing in transsexual women. Arch Sex Behav 2012;41:1069-71.

15. Hamada A, Kingsberg S, Wierckx K, et al. Semen characteristics of transwomen referred for sperm banking before sex transition: a case series. Andrologia 2015;47:832-8.

16. Li K, Rodriguez D, Gabrielsen JS, et al. Sperm cryopreservation of transgender individuals: trends and findings in the past decade. Andrology 2018;6:860-4.

17. Adeleye AJ, Reid G, Kao CN, et al. Semen Parameters Among Transgender Women With a History of Hormonal Treatment. Urology 2019;124:136-41.

18. Schneider F, Neuhaus N, Wistuba J, et al. Testicular Functions and Clinical Characterization of Patients with Gender Dysphoria (GD) Undergoing Sex Reassignment Surgery (SRS). J Sex Med 2015;12:2190-200.

19. Martini F, Nath JL. Anatomy \& physiology. 2nd ed. San Francisco: Benjamin Cummings, 2010.

20. Wallace SA, Blough KL, Kondapalli LA. Fertility preservation in the transgender patient: expanding oncofertility care beyond cancer. Gynecol Endocrinol 2014;30:868-71.

21. Mattawanon N, Spencer JB, Schirmer DA, 3rd, et al. Fertility preservation options in transgender people: A review. Rev Endocr Metab Disord 2018;19:231-42. 
22. Sato T, Katagiri K, Yokonishi T, et al. In vitro production of fertile sperm from murine spermatogonial stem cell lines. Nat Commun 2011;2:472.

23. Duggal G, Heindryckx B, Deroo T, et al. Use of pluripotent stem cells for reproductive medicine: are we there yet? Vet Q 2014;34:42-51.

24. Garcia MM, Ohta AT, Walsh TJ, et al. A noninvasive, motility independent, sperm sorting method and technology to identify and retrieve individual viable nonmotile sperm for intracytoplasmic sperm injection. J Urol 2010;184:2466-72.

25. Reefhuis J, Honein MA, Schieve LA, et al. Assisted reproductive technology and major structural birth defects in the United States. Hum Reprod 2009;24:360-6.

26. Horák S, Olejek A, Widlak P. Sperm DNA adducts impair fertilization during ICSI but not during IVF. Folia Histochem Cytobiol 2007;45 Suppl 1:S99-104.

27. Brännström $M$, Johannesson L, Bokstrom $\mathrm{H}$, et al. Livebirth after uterus transplantation. Lancet 2015;385:607-16.

28. Alghrani A. Uterus transplantation in and beyond cisgender women: revisiting procreative liberty in light of emerging reproductive technologies. J Law Biosci 2018;5:301-28.

29. Jones BP, Williams NJ, Saso S, et al. Uterine transplantation in transgender women. BJOG 2019;126:152-6.

30. Nahata L, Chen D, Moravek MB, et al. Understudied and Under-Reported: Fertility Issues in Transgender Youth-A Narrative Review. J Pediatr 2019;205:265-71.

31. Armuand G, Dhejne C, Olofsson JI, et al. Transgender men's experiences of fertility preservation: a qualitative study. Hum Reprod 2017;32:383-90.

32. Light AD, Obedin-Maliver J, Sevelius JM, et al. Transgender men who experienced pregnancy after female-to-male gender transitioning. Obstet Gynecol 2014;124:1120-7.

33. Nakamura A, Watanabe $M$, Sugimoto $M$, et al. Doseresponse analysis of testosterone replacement therapy in patients with female to male gender identity disorder. Endocr J 2013;60:275-81.

34. Steinle K. Hormonal management of the female-tomale transgender patient. J Midwifery Womens Health 2011;56:293-302.

35. Broughton D, Omurtag K. Care of the transgender or gender-nonconforming patient undergoing in vitro fertilization. Int J Transgenderism 2017;18:372-5.

36. Maxwell S, Noyes N, Keefe D, et al. Pregnancy Outcomes
After Fertility Preservation in Transgender Men. Obstet Gynecol 2017;129:1031-4.

37. De Roo C, Lierman S, Tilleman K, et al. Ovarian tissue cryopreservation in female-to-male transgender people: insights into ovarian histology and physiology after prolonged androgen treatment. Reprod Biomed Online 2017;34:557-66.

38. Beckmann MW, Dittrich R, Lotz L, et al. Fertility protection: complications of surgery and results of removal and transplantation of ovarian tissue. Reprod Biomed Online 2018;36:188-96.

39. Donnez J, Dolmans MM, Pellicer A, et al. Restoration of ovarian activity and pregnancy after transplantation of cryopreserved ovarian tissue: a review of 60 cases of reimplantation. Fertil Steril 2013;99:1503-13.

40. Jensen CF, Ohl DA, Parker WR, et al. Optimizing human semen cryopreservation by reducing test vial volume and repetitive test vial sampling. Fertil Steril 2015;103:640-6.e1.

41. Jadoul P, Guilmain A, Squifflet J, et al. Efficacy of ovarian tissue cryopreservation for fertility preservation: lessons learned from 545 cases. Hum Reprod 2017;32:1046-54.

42. Abir R, Ben-Aharon I, Garor R, et al. Cryopreservation of in vitro matured oocytes in addition to ovarian tissue freezing for fertility preservation in paediatric female cancer patients before and after cancer therapy. Hum Reprod 2016;31:750-62.

43. Segers I, Mateizel I, Van Moer E, et al. In vitro maturation (IVM) of oocytes recovered from ovariectomy specimens in the laboratory: a promising "ex vivo" method of oocyte cryopreservation resulting in the first report of an ongoing pregnancy in Europe. J Assist Reprod Genet 2015;32:1221-31.

44. Practice Committees of the American Society for Reproductive Medicine and the Society for Assisted Reproductive Technology. In vitro maturation: a committee opinion. Fertil Steril 2013;99:663-6.

45. Tornello SL, Bos H. Parenting Intentions Among Transgender Individuals. LGBT Health 2017;4:115-20.

46. Riggs DW, Bartholomaeus C. Fertility preservation decision making amongst Australian transgender and nonbinary adults. Reprod Health 2018;15:181.

47. Pyne J, Bauer G, Bradley K. Transphobia and other stressors impacting trans parents. J GLBT Fam Stud 2015:107-26.

48. Auer MK, Fuss J, Nieder TO, et al. Desire to Have Children Among Transgender People in Germany: A Cross-Sectional Multi-Center Study. J Sex Med 
2018;15:757-67.

49. Grant JM, Mottet LA, Tanis J, et al. Injustice at every turn: a report of the national transgender discrimination survey. Washington, DC: National Center for Transgender Equality and National Gay and Lesbian Task Force, 2011.

50. Ross LE, Epstein R, Anderson S, et al. Policy, practice, and personal narratives: Experiences of LGBTQ people with adoption in Ontario, Canada. Adoption Quarterly 2009.

51. James-Abra S, Tarasoff LA, Green D, et al. Trans people's experiences with assisted reproduction services: a qualitative study. Hum Reprod 2015;30:1365-74.

52. Chen D, Matson M, Macapagal K, et al. Attitudes Toward Fertility and Reproductive Health Among Transgender and Gender-Nonconforming Adolescents. J Adolesc Health 2018;63:62-8.

53. Wu HY, Yin O, Monseur B, et al. Lesbian, gay, bisexual, transgender content on reproductive endocrinology and infertility clinic websites. Fertil Steril 2017;108:183-91.

54. Tishelman AC, Sutter ME, Chen D, et al. Health care provider perceptions of fertility preservation barriers and challenges with transgender patients and families: qualitative responses to an international survey. J Assist Reprod Genet 2019;36:579-88.

55. Chen D, Kolbuck VD, Sutter ME, et al. Knowledge, Practice Behaviors, and Perceived Barriers to Fertility Care Among Providers of Transgender Healthcare. J Adolesc Health 2019;64:226-34.

56. Johnson EK, Finlayson C. Preservation of Fertility Potential for Gender and Sex Diverse Individuals. Transgend Health 2016;1:41-4.

57. Quinn GP, Vadaparampil ST, Lee JH, et al. Physician referral for fertility preservation in oncology patients: a national study of practice behaviors. J Clin Oncol 2009;27:5952-7.

Cite this article as: Sterling J, Garcia MM. Fertility preservation options for transgender individuals. Transl Androl Urol 2020;9(Suppl 2):S215-S226. doi: 10.21037/tau.2019.09.28
58. Quinn GP, Vadaparampil ST, Fertility Preservation Research G. Fertility preservation and adolescent/young adult cancer patients: physician communication challenges. J Adolesc Health 2009;44:394-400.

59. Klosky JL, Anderson LE, Russell KM, et al. Provider Influences on Sperm Banking Outcomes Among Adolescent Males Newly Diagnosed With Cancer. J Adolesc Health 2017;60:277-83.

60. Shnorhavorian M, Kroon L, Jeffries H, et al. Creating a standardized process to offer the standard of care: continuous process improvement methodology is associated with increased rates of sperm cryopreservation among adolescent and young adult males with cancer. J Pediatr Hematol Oncol 2012;34:e315-9.

61. Ohta AT, Garcia M, Valley JK, et al. Motile and nonmotile sperm diagnostic manipulation using optoelectronic tweezers. Lab Chip 2010;10:3213-7.

62. Valley JK, Swinton P, Boscardin WJ, et al. Preimplantation mouse embryo selection guided by light-induced dielectrophoresis. PLoS One 2010;5:e10160.

63. Lewis SE, Aitken RJ. DNA damage to spermatozoa has impacts on fertilization and pregnancy. Cell Tissue Res 2005;322:33-41.

64. Society for Assisted Reproductive Technology Facts and Figures 2019; Available online: https://www.sart.org/ patients/history-of-ivf/

65. Lewis SE, Agbaje I, Alvarez J. Sperm DNA tests as useful adjuncts to semen analysis. Syst Biol Reprod Med 2008;54:111-25.

66. Varghese AC, Goldberg E, Agarwal A. Current and future perspectives on intracytoplasmic sperm injection: a critical commentary. Reprod Biomed Online 2007;15:719-27.

67. Ahmadi A, Ng SC. Fertilizing ability of DNA-damaged spermatozoa. J Exp Zool 1999;284:696-704. 